\title{
Acceleration of Augmented EFIE Using Multilevel Complex Source Beam Method
}

\author{
Lianning Song, Yongpin Chen, Ming Jiang, Jun Hu, and Zaiping Nie \\ Department of Microwave Engineering, University of Electronic Science and Technology of China, Chengdu, Sichuan 610054, China \\ Correspondence should be addressed to Jun Hu; hujun@uestc.edu.cn
}

Received 20 April 2017; Accepted 12 June 2017; Published 13 July 2017

Academic Editor: Song Guo

Copyright (C) 2017 Lianning Song et al. This is an open access article distributed under the Creative Commons Attribution License, which permits unrestricted use, distribution, and reproduction in any medium, provided the original work is properly cited.

\begin{abstract}
The computation of the augmented electric field integral equation (A-EFIE) is accelerated by using the multilevel complex source beam (MLCSB) method. As an effective solution of the low-frequency problem, A-EFIE includes both current and charge as unknowns to avoid the imbalance between the vector potentials and the scalar potentials in the conventional EFIE. However, dense impedance submatrices are involved in the A-EFIE system, and the computational cost becomes extremely high for problems with a large number of unknowns. As an exact solution to Maxwell's equations, the complex source beam (CSB) method can be well tailored for A-EFIE to accelerate the matrix-vector products in an iterative solver. Different from the commonly used multilevel fast multipole algorithm (MLFMA), the CSB method is free from the problem of low-frequency breakdown. In our implementation, the expansion operators of CSB are first derived for the vector potentials and the scalar potentials. Consequently, the aggregation and disaggregation operators are introduced to form a multilevel algorithm to reduce the computational complexity. The accuracy and efficiency of the proposed method are discussed in detail through a variety of numerical examples. It is observed that the numerical error of the MLCSB-AEFIE keeps constant for a broad frequency range, indicating the good stability and scalability of the proposed method.
\end{abstract}

\section{Introduction}

The method of moments (MoM) [1] for solving electric field integral equation (EFIE) has received intensive study in the analysis of electromagnetic (EM) radiation, scattering, and circuit problems in recent years. However, the commonly used EFIE with RWG basis function suffers from a "lowfrequency breakdown" problem [2] when the frequency decreases and/or when the mesh is refined. In this situation, EFIE is dominated by the scalar potentials while the contribution from the vector potentials is overwhelmed due to the finite machine precision. Since the scalar potential term is singular, the EFIE matrix system becomes extremely ill-conditioned and results in convergence issue when the iterative solver is applied.

To address this difficulty, several methods have been proposed in the past years. A widely used remedy is the loop-tree or loop-star decomposition [3] which decouples the magneto- and electrostatic physics. However, the extraction of global loops is difficult for complex interconnecting structures, and the system matrix becomes ill-conditioned as the frequency increases. On the other hand, the convergence issue can be improved by a Calderón preconditioner [4] when iterative solvers are applied. To avoid the involved loop searching process, alternative formulations have been proposed by separating current and charge, such as the current and charge integral equation (CCIE) [5], split potential integral equation (SPIE) [6,7], and augmented electric field integral equation (A-EFIE) [8-11]. In the A-EFIE method, the contributions of the vector potentials and the scalar potentials are separated by adding charge as extra unknowns, where the current continuity equation is explicitly enforced. Frequency scaling is then implemented to stabilize the system equation. The resulting impendence matrix of A-EFIE has the characteristics of a saddle point matrix and is wellconditioned at low frequencies. Recently, the perturbation method is introduced to enhance the accuracy of A-EFIE at extremely low frequencies [12]. The augmented equivalence principle algorithm (A-EPA) [13] and discontinuous Galerkin (DG) method [14] are combined with the A-EFIE for domain decomposition problems. 
As the number of unknowns increases, a fast algorithm has to be incorporated into the iterative solver to reduce the operation complexity of the matrix-vector product (MVP). Such fast algorithms should be low-frequency stable; for instance, the low-frequency multilevel fast multipole algorithm (LF-MLFMA) [15], the multilevel accelerated Cartesian expansion algorithm (MLACEA) [16], the multilevel adaptive cross-approximation (MLACA) algorithm [17], and fast Fourier transform (FFT) [18] are eligible candidates for such purpose. Recently, a complex source beam-method of moment (CSB-MoM) is proposed to accelerate the far-field interactions of MoM at midfrequencies [19]. The object is first divided into groups and complex source beams (CSBs) are used to expand the fields of the basis functions residing in each group [20,21]. A multilevel version of this method is developed in [22]. To further improve the computational efficiency, a nested complex source beam (NCSB) method is proposed by utilizing an equivalent relationship between adjacent levels [23]. Since the CSBs are exact solutions of Maxwell's equations, any arbitrary EM fields can be expanded in terms of a set of CSBs $[20,24]$. Therefore, this method can be extended to solve the low-frequency problems without any theoretical barriers.

This paper is organized as follows. The basis formulations of CSB-MoM and A-EFIE are briefly reviewed in Section 2. In Section 3, the CSB-MoM is integrated into the A-EFIE system to remedy the low-frequency breakdown problem. The detailed derivation of the CSB expansions for A-EFIE is first presented. The aggregation/disaggregation, translation operators for a multilevel CSB method are then discussed. Finally, numerical examples are summarized in Section 4 to demonstrate the validity and efficiency of the proposed method.

\section{Theory Background}

Given a 3D perfectly electrical conducting (PEC) body defined by its surface, the conventional MoM formulation can be applied to the electric field integral equation (EFIE), leading to a matrix equation of the form

$$
\mathbf{Z j}=\mathbf{b}
$$

where $\mathbf{j}$ is the unknown vector for the surface current density, $\mathbf{b}$ is the excitation vector, and $\mathbf{Z}$ is the dense impedance matrix. The impedance matrix can be expressed as

$$
\mathbf{Z}_{i j}=\int_{S_{i}} \mathbf{f}_{i}(\mathbf{r}) \cdot \int_{S_{j}} \overline{\bar{G}}\left(\mathbf{r}, \mathbf{r}^{\prime}\right) \mathbf{f}_{j}\left(\mathbf{r}^{\prime}\right) d S^{\prime},
$$

where

$$
\overline{\bar{G}}\left(\mathbf{r}, \mathbf{r}^{\prime}\right)=\left[\overline{\bar{I}}+\frac{\nabla \nabla}{k_{0}^{2}}\right] g\left(\mathbf{r}, \mathbf{r}^{\prime}\right)
$$

is the dyadic Green function and $g\left(\mathbf{r}, \mathbf{r}^{\prime}\right)=e^{i k_{0}\left|\mathbf{r}-\mathbf{r}^{\prime}\right|} / 4 \pi\left|\mathbf{r}-\mathbf{r}^{\prime}\right|$ is the scalar Green function. Moreover, $\mathbf{f}_{j}\left(\mathbf{r}^{\prime}\right)$ and $\mathbf{f}_{i}(\mathbf{r})$ denote the RWG basis functions for expanding surface current and Galerkin testing, respectively. This equation suffers from the low-frequency breakdown problem, because the contribution of the vector potential is swamped by that of the scalar potential at low frequencies due to the finite machine precision.

2.1. Formulation of CSB-MoM for EFIE. In CSB-MoM method, the MVP acceleration of $\mathbf{Z j}=\mathbf{b}$ is achieved through a FMM-like near-field and far-field decomposition. For the far-field interactions $\mathbf{Z}_{F} \mathbf{j}$ between well separated groups, the MVP are carried out by a series of CSBs launched on a complex equivalence surface enclosing each group:

$$
\mathbf{Z}_{F} \mathbf{j}=\mathbf{Z}_{m m^{\prime}} \mathbf{j}_{m^{\prime}}=\sum_{\varepsilon, \varepsilon^{\prime}=\theta, \phi}\left(\mathbf{W}_{m}^{\varepsilon}\right)^{T} \mathbf{T}_{L, m m^{\prime}}^{\varepsilon \varepsilon^{\prime}} \mathbf{W}_{m^{\prime}}^{\varepsilon^{\prime}} \mathbf{j}_{m^{\prime}},
$$

where $m$ and $m^{\prime}$ denote the observation and source groups and $L$ indicates the finest level (single level in CSB-MoM). $\mathbf{W}_{m^{\prime}}^{\varepsilon}$ and $\mathbf{W}_{m}^{\varepsilon^{\prime}}$ are the expansion matrices for both $\theta$ and $\phi$ components, which can be obtained by the far-field matching of basis functions in groups $m$ and $m^{\prime}$, respectively [19]. Superscript $T$ stands for the transpose of the corresponding matrix. It needs to be emphasized that only $\theta$ and $\phi$ components are considered in CSBs, since the far-field radiation fields contain only $\theta$ and $\phi$ components.

By using the expansion matrices, the CSB expansion coefficients for the source group $m^{\prime}$ can be expanded from the surface currents as

$$
\mathbf{s}_{L, m^{\prime}}=\left[\begin{array}{c}
\mathbf{s}_{L, m^{\prime}}^{\theta} \\
\mathbf{s}_{L, m^{\prime}}^{\phi}
\end{array}\right]=\left[\begin{array}{c}
\mathbf{W}_{m^{\prime}}^{\theta} \\
\mathbf{W}_{m^{\prime}}^{\phi}
\end{array}\right] \mathbf{j}_{m^{\prime}} .
$$

$\mathbf{T}_{L, m m^{\prime}}^{\varepsilon \varepsilon^{\prime}}$ in (4) is the translation matrix, of which the elements are expressed as

$$
\left[\mathbf{T}_{L, m m^{\prime}}^{\varepsilon \varepsilon^{\prime}}\right]_{q q^{\prime}}=\widehat{\varepsilon}_{q} \cdot \overline{\bar{G}}\left(\widetilde{\mathbf{r}}_{L, m}^{q}, \widetilde{\mathbf{r}}_{L, m^{\prime}}^{q^{\prime}}\right) \cdot \widehat{\varepsilon}_{q^{\prime}}^{\prime}
$$

where $\widehat{\varepsilon}_{q}$ and $\widehat{\varepsilon}_{q^{\prime}}^{\prime}$ denote the unit vectors of $q$ th and $q^{\prime}$ th CSB, respectively. The complex position vectors $\widetilde{\mathbf{r}}_{L, m}^{q}$ and $\widetilde{\mathbf{r}}_{L, m^{\prime}}^{\prime q^{\prime}}$ are the launch points of CSBs associated with groups $m$ and $m^{\prime} \cdot \overline{\bar{G}}\left(\widetilde{\mathbf{r}}_{L, m}^{q}, \widetilde{\mathbf{r}}_{L, m^{\prime}}^{\prime q^{\prime}}\right)$ is the dyadic Green function with complex arguments. The directional property of CSBs can be used to reduce the computational cost in the translation procedure.

2.2. Formulation of $A$-EFIE. In the augmented electric field integral equation, the surface current is discretized by the normalized RWG basis function, which is modified by removing the length of the common edge

$$
\boldsymbol{\rho}_{i}(\mathbf{r})= \begin{cases}\frac{\mathbf{r}-\mathbf{r}_{i}^{+}}{2 A_{i}^{+}}, & \mathbf{r} \in T_{i}^{+} \\ \frac{\mathbf{r}_{i}^{-}-\mathbf{r}}{2 A_{i}^{-}}, & \mathbf{r} \in T_{i}^{-} \\ 0, & \text { otherwise }\end{cases}
$$

where $A_{i}^{ \pm}$are the area of triangles $T_{i}^{ \pm}, \mathbf{r}_{i}^{ \pm}$are the free vertices of the two triangles. On the other hand, the surface charge 
density is approximated by the pulse function defined on each triangle, which is expressed as

$$
h_{i}(\mathbf{r})= \begin{cases}\frac{1}{A_{i}}, & \mathbf{r} \in T_{i} \\ 0, & \text { otherwise. }\end{cases}
$$

Combining EFIE in (1) and the current continuity condition between current and charge, we can arrive at the A-EFIE equation

$$
\left[\begin{array}{cc}
\overline{\mathbf{V}} & \overline{\mathbf{D}}^{T} \cdot \overline{\mathbf{P}} \\
\overline{\mathbf{D}} & k_{0}^{2} \overline{\mathbf{I}}
\end{array}\right] \cdot\left[\begin{array}{l}
i k_{0} \mathbf{j} \\
c_{0} \phi
\end{array}\right]=\left[\begin{array}{c}
\eta_{0}^{-1} \mathbf{b} \\
0
\end{array}\right]
$$

with

$$
\begin{aligned}
& \overline{\mathbf{V}}_{i j}=\int_{S_{i}} \boldsymbol{\rho}_{i}(\mathbf{r}) \cdot \int_{S_{j}} g\left(\mathbf{r}, \mathbf{r}^{\prime}\right) \boldsymbol{\rho}_{j}\left(\mathbf{r}^{\prime}\right) d S^{\prime} d S \\
& \overline{\mathbf{P}}_{i j}=\int_{S_{i}} h_{i}(\mathbf{r}) \cdot \int_{S_{j}} g\left(\mathbf{r}, \mathbf{r}^{\prime}\right) h_{j}\left(\mathbf{r}^{\prime}\right) d S^{\prime} d S \\
& \mathbf{b}_{i}=\int_{S_{i}} \mathbf{f}_{i}(\mathbf{r}) \cdot \mathbf{E}^{i} d S \\
& \overline{\mathbf{D}}_{i j}= \begin{cases}1, & T_{i} \in S_{j}, \text { the positive part } \\
-1, & T_{i} \in S_{j}, \text { the negative part } \\
0, & T_{i} \notin S_{j},\end{cases}
\end{aligned}
$$

where $\mathbf{j}$ and $\phi$ denote the unknown coefficients for current and charge, $k_{0}$ and $\eta_{0}$ are the wave number and the wave impedance in free space, and $c_{0}$ is the light speed in free space. The dense matrix $\overline{\mathbf{V}}$ represents the vector potentials and depicts the current interactions between inner edges. On the other hand, $\overline{\mathbf{P}}$ denotes the scalar potentials, which describes charges interactions between triangles. The sparse matrix $\overline{\mathbf{D}}$ represents the relationship between edge and the triangles patch, and $\overline{\mathbf{I}}$ is the identity matrix. In the matrix system (9), the vector potential and scalar potential are balanced by using a proper frequency scaling, which is critical for low-frequency problems.

\section{Implementation of MLCSB for A-EFIE}

3.1. CSB Expansions for A-EFIE. Motivated by the idea of CSB expansion method for EFIE, we can derive the expansions for A-EFIE in a similar way. The inner integrals of (10) and (11) can be written as

$$
\begin{aligned}
& \mathbf{V}_{i}(\mathbf{r})=\int_{S_{i}} g\left(\mathbf{r}, \mathbf{r}^{\prime}\right) \boldsymbol{\rho}_{i}\left(\mathbf{r}^{\prime}\right) d S^{\prime} \\
& \mathbf{P}_{j}(\mathbf{r})=\int_{S_{j}} g\left(\mathbf{r}, \mathbf{r}^{\prime}\right) h_{j}\left(\mathbf{r}^{\prime}\right) d S^{\prime},
\end{aligned}
$$

where $i$ and $j$ represent the $i$ th normalized RWG basis function and the $j$ th pulse function in group $m^{\prime}$. The above integral equation is equivalent to the summation of CSB vector and scalar potentials:

$$
\begin{aligned}
& \mathbf{V}_{i}(\mathbf{r})=\sum_{q=1}^{Q} g\left(\mathbf{r}, \widetilde{\mathbf{r}}_{q}^{\prime}\right)\left(\widehat{x} w_{q, i}^{x}+\widehat{y} w_{q, i}^{y}+\widehat{z} w_{q, i}^{z}\right) \\
& \mathbf{P}_{j}(\mathbf{r})=\sum_{q=1}^{Q} g\left(\mathbf{r}, \widetilde{\mathbf{r}}_{q}^{\prime}\right) w_{q, j}^{p},
\end{aligned}
$$

where $\widehat{x} w_{q, i}^{x}+\widehat{y} w_{q, i}^{y}+\widehat{z} w_{q, i}^{z}$ is the vector CSB weight for current $i, w_{q, j}^{p}$ is the weight for charge $j$, and $Q$ represents the total number of beams in this discretization. Combining (16) with (14) and (17) with (15) and testing on a far-field matching point, we get

$$
\begin{gathered}
\widehat{x} \cdot \sum_{q=1}^{Q} g\left(\mathbf{r}_{t}, \widetilde{\mathbf{r}}_{q}^{\prime}\right) \widehat{x} w_{q, i}^{x}=\widehat{x} \cdot \int_{S_{i}} g\left(\mathbf{r}_{t}, \mathbf{r}^{\prime}\right) \boldsymbol{\rho}_{i}\left(\mathbf{r}^{\prime}\right) d S^{\prime} \\
\widehat{y} \cdot \sum_{q=1}^{Q} g\left(\mathbf{r}_{t}, \widetilde{\mathbf{r}}_{q}^{\prime}\right) \hat{y} w_{q, i}^{y}=\widehat{y} \cdot \int_{S_{i}} g\left(\mathbf{r}_{t}, \mathbf{r}^{\prime}\right) \boldsymbol{\rho}_{i}\left(\mathbf{r}^{\prime}\right) d S^{\prime} \\
\widehat{z} \cdot \sum_{q=1}^{Q} g\left(\mathbf{r}_{t}, \widetilde{\mathbf{r}}_{q}^{\prime}\right) \widehat{z} w_{q, i}^{z}=\widehat{z} \cdot \int_{S_{i}} g\left(\mathbf{r}_{t}, \mathbf{r}^{\prime}\right) \boldsymbol{\rho}_{i}\left(\mathbf{r}^{\prime}\right) d S^{\prime} \\
\sum_{q=1}^{Q} g\left(\mathbf{r}_{t}, \widetilde{\mathbf{r}}_{q}^{\prime}\right) w_{q, j}^{p}=\int_{S_{j}} g\left(\mathbf{r}_{t}, \mathbf{r}^{\prime}\right) h_{j}\left(\mathbf{r}^{\prime}\right) d S^{\prime},
\end{gathered}
$$

where $\mathbf{r}_{t}(t=1,2, \ldots, Q)$ is the matching point. Those linear equations can be converted into a matrix form and combined as a multiple right-hand side problem by all the current and charge basis in the group $m^{\prime}$ :

$$
\mathbf{G}\left[\mathbf{W}_{m^{\prime}}^{x}, \mathbf{W}_{m^{\prime}}^{y}, \mathbf{W}_{m^{\prime}}^{z}, \mathbf{W}_{m^{\prime}}^{p}\right]=\left[\mathbf{V}_{m^{\prime}}^{x}, \mathbf{V}_{m^{\prime}}^{y}, \mathbf{V}_{m^{\prime}}^{z}, \mathbf{P}_{m^{\prime}}\right],
$$

where

$$
\begin{aligned}
{[\mathbf{G}]_{t q} } & =g\left(\mathbf{r}_{t}, \widetilde{\mathbf{r}}_{q}^{\prime}\right) \\
{\left[\mathbf{W}_{m^{\prime}}^{v}\right]_{q i} } & =w_{q, i}^{v} \quad(v=x, y, z) \\
{\left[\mathbf{W}_{m^{\prime}}^{p}\right]_{q j} } & =w_{q, j}^{p} \\
{\left[\mathbf{V}_{m^{\prime}}^{v}\right]_{t i} } & =\widehat{v}_{t} \cdot \int_{S_{i}} g\left(\mathbf{r}_{t}, \mathbf{r}^{\prime}\right) \boldsymbol{\rho}_{i}\left(\mathbf{r}^{\prime}\right) d S^{\prime} \quad(v=x, y, z) \\
{\left[\mathbf{P}_{m^{\prime}}\right]_{t j} } & =\int_{S_{j}} g\left(\mathbf{r}_{t}, \mathbf{r}^{\prime}\right) h_{j}\left(\mathbf{r}^{\prime}\right) d S^{\prime} .
\end{aligned}
$$

By using the expansion matrices $\mathbf{W}_{m^{\prime}}^{x}, \mathbf{W}_{m^{\prime}}^{y}, \mathbf{W}_{m^{\prime}}^{z}$, and $\mathbf{W}_{m^{\prime}}^{p}$, the CSB expansion coefficients for vector potentials of group $m^{\prime}$ can be expanded from $\mathbf{j}_{m^{\prime}}$ as

$$
\overline{\mathbf{s}}_{L, m^{\prime}}^{v}=\left[\mathbf{s}_{L, m^{\prime}}^{x}, \mathbf{s}_{L, m^{\prime}}^{y}, \mathbf{s}_{L, m^{\prime}}^{z}\right]=\left[\mathbf{W}_{m^{\prime}}^{x}, \mathbf{W}_{m^{\prime}}^{y}, \mathbf{W}_{m^{\prime}}^{z}\right] \mathbf{j}_{m^{\prime}} .
$$

Similarly, the coefficients for scalar ones are

$$
\mathbf{s}_{L, m^{\prime}}^{p}=\mathbf{W}_{m^{\prime}}^{p} \phi_{m^{\prime}}
$$


Hence, the far-field MVP procedure for (10) and (11) can be obtained with a similar process in (4):

$$
\begin{aligned}
& \overline{\mathbf{V}}_{m m^{\prime}} \mathbf{j}_{m^{\prime}}=\sum_{v=x, y, z}\left(\mathbf{W}_{m}^{v}\right)^{T} \mathbf{T}_{L, m m^{\prime}} \mathbf{W}_{m^{\prime}}^{v} \mathbf{j}_{m^{\prime}} \\
& \overline{\mathbf{P}}_{m m^{\prime}} \phi_{m^{\prime}}=\left(\mathbf{W}_{m}^{d}\right)^{T} \mathbf{T}_{L, m m^{\prime}} \mathbf{W}_{m^{\prime}}^{p} \phi_{m^{\prime}},
\end{aligned}
$$

where the translation operator $\mathbf{T}_{L, m m^{\prime}}$ is expressed as

$$
\left[\mathbf{T}_{L, m m^{\prime}}\right]_{q q^{\prime}}=g\left(\widetilde{\mathbf{r}}_{L, m}^{q}, \widetilde{\mathbf{r}}_{L, m^{\prime}}^{\prime q^{\prime}}\right) .
$$

It is noticed that the scalar Green function with complex arguments is used here, and the translation for the vector and scalar expansion coefficients shares the same operators.

3.2. Multilevel Algorithm of CSB for A-EFIE. So far, we have derived the CSB expansion method for A-EFIE and have presented the far-group MVP process with the help of translation operators in a single level algorithm. In the following, we will obtain the aggregation and disaggregation operators of CSB expansion coefficients for both (10) and (11), to realize a multilevel algorithm. Firstly, the vector and scalar potentials by the $q_{l+1}$ th CSB in a group is

$$
\begin{aligned}
& \mathbf{V}_{q_{l+1}}(\mathbf{r})=g\left(\mathbf{r}, \widetilde{\mathbf{r}}_{q_{l+1}}^{\prime}\right)\left(\widehat{x} s_{q_{l+1}}^{x}+\widehat{y} s_{q_{l+1}}^{y}+\widehat{z} s_{q_{l+1}}^{z}\right) \\
& \mathbf{P}_{q_{l+1}}(\mathbf{r})=g\left(\mathbf{r}, \widetilde{\mathbf{r}}_{q_{l+1}}^{\prime}\right) s_{q_{l+1}}^{p}
\end{aligned}
$$

where $l+1$ means the $(l+1)$ th level of the octree in the multilevel algorithm. By applying the summation and testing procedures similar to (16)-(18), a linear system can be set up to obtain the equivalent relationship of CSBs between two adjacent levels:

$$
\mathbf{G}_{l} \mathbf{A}_{l}=\mathbf{P}_{l+1},
$$

where $\left[\mathbf{G}_{l}\right]_{t_{l} q_{l}}=g\left(\mathbf{r}_{t_{l}}, \widetilde{\mathbf{r}}_{q_{l}}^{\prime}\right)$ is the matching matrix in parent level $l$. This matching matrix connects the equivalent CSB sources to the scalar potentials $\mathbf{P}_{l+1}$. Different from the righthand side in (19), the right-hand side here for each vector potential component is the same as the scalar one, which is

$$
\left[\mathbf{P}_{l+1}\right]_{q_{l} q_{l+1}}=g\left(\mathbf{r}_{q_{l}} \mid \tilde{\mathbf{r}}_{q_{l+1}}^{\prime}\right) .
$$

Once $\mathbf{G}_{l}$ and $\mathbf{P}_{l+1}$ are assembled, the aggregation matrix $\mathbf{A}_{l}$ for level $l$ can be numerically solved. By using the aggregation matrix, the CSB expansion coefficients of the parent group in level $l$ can be obtained efficiently from its child groups in level $l+1$ :

$$
\begin{aligned}
& \overline{\mathbf{s}}_{l, m^{\prime}}^{v}=\left[\mathbf{s}_{l, m^{\prime}}^{x}, \mathbf{s}_{l, m^{\prime}}^{y}, \mathbf{s}_{l, m^{\prime}}^{z}\right]=\sum_{n^{\prime} \in \operatorname{child}\left(m^{\prime}\right)} \mathbf{A}_{l} \overline{\mathbf{s}}_{l+1, n^{\prime}}^{v} \\
& \mathbf{s}_{l, m^{\prime}}^{p}=\sum_{n^{\prime} \in \operatorname{child}\left(m^{\prime}\right)} \mathbf{A}_{l} \mathbf{s}_{l+1, n^{\prime}}^{p} .
\end{aligned}
$$

Similar to the multilevel fast multipole algorithm (MLFMA) [25], the CSB expansion coefficients of a receiving group in level $l+1$ are obtained from the translation in the same level as well as the disaggregation from its parent level l. The disaggregation matrix can be easily obtained from the transpose of the aggregation matrices.

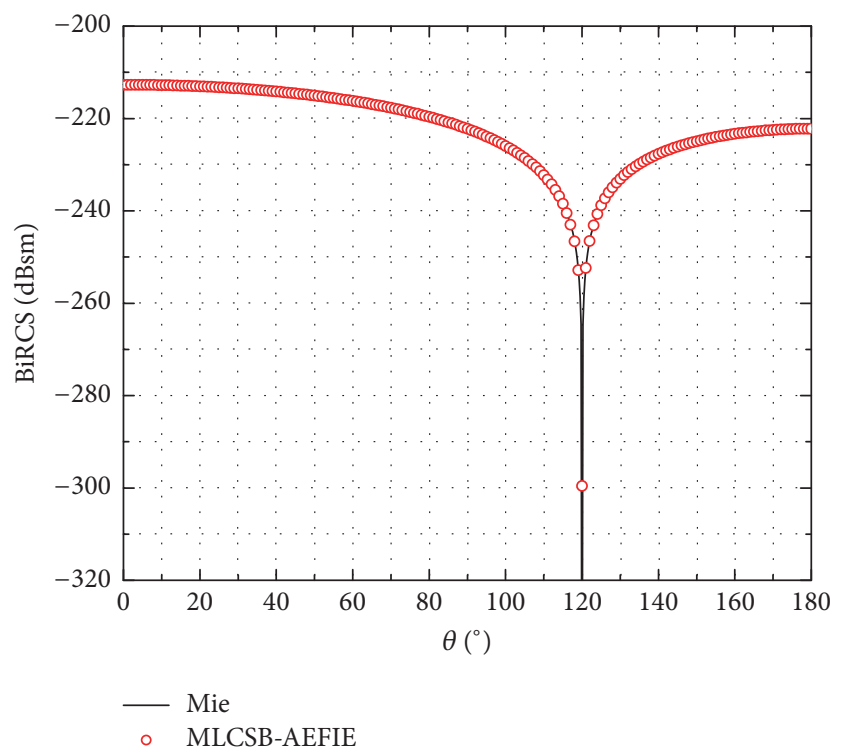

FIGURE 1: Bistatic RCS of the PEC sphere at $100 \mathrm{~Hz}$, validated by the Mie series.

\section{Numerical Results}

In this section, the accuracy, error analysis, computational complexity, and the efficiency of the method are investigated through several numerical examples. All the examples were run on a computer of 2 processors, each with 14 cores at 2.6 GHz, $512 \mathrm{~GB}$ memory, and OpenMP parallelization.

4.1. Small Sphere. To show the accuracy of MLCSB-AEFIE at low frequencies, the electromagnetic scattering by a PEC sphere of $1 \mathrm{~m}$ radius is analyzed at $100 \mathrm{~Hz}$. The sphere is discretized with 1,764 triangular patches, which corresponds to 2,646 inner edges. A three levels' MLCSB algorithm is used with a group size of $8.3 \times 10^{-8} \lambda$ at the finest level. The incident angle of a plane wave is $\theta_{i}=0^{\circ}, \varphi_{i}=0^{\circ}$, and the observed azimuth angle is fixed at $\theta_{i}=0^{\circ}$. The residual error threshold is set to be $10^{-15}$ for GMRES-30. It takes 80 iteration steps to converge, with the help of the saddle point preconditioner in [9]. A good agreement of the bistatic RCS is observed in Figure 1, as compared with the analytical solution of Mie series.

4.2. Computational Complexity. To demonstrate the computational complexity of the proposed method, the plane wave scattering of a PEC cube with a side length of $0.1 \mathrm{~m}$ is calculated at $300 \mathrm{MHz}$. The electric size of the cube is $0.173 \lambda$. The surface of the cube is discretized into six different meshes: Mesh A, Mesh B, Mesh C, Mesh D, Mesh E, and Mesh F. The coarsest Mesh A has 1262 planar triangles and 1893 interior edges, and the average edge length is $1.05 \times 10^{-2} \lambda$. Then we refine Mesh A by halving the edge length recursively until Mesh F. Mesh F comprises 1,534,536 planar triangles and 2,301,804 interior edges, and the average edge length is $3 \times 10^{-4} \lambda$. 
TABLE 1: Computational statistics of the PEC cube with different meshes at $300 \mathrm{MHz}$.

\begin{tabular}{lcccccccccc}
\hline Mesh & $\begin{array}{c}\text { Number of } \\
\text { RWG }\end{array}$ & $\begin{array}{c}\text { Number of } \\
\text { triangles }\end{array}$ & $\begin{array}{c}\text { Near-field } \\
\text { Mem. } \\
(\mathrm{MB})\end{array}$ & $\begin{array}{c}\text { Expansion } \\
\text { Mem. } \\
(\mathrm{MB})\end{array}$ & $\begin{array}{c}\text { Translation } \\
\text { Mem. (MB) }\end{array}$ & $\begin{array}{c}\text { Interpolation } \\
\text { Mem. }(\mathrm{MB})\end{array}$ & $\begin{array}{c}\text { Total } \\
\text { Mem. } \\
(\mathrm{MB})\end{array}$ & $\begin{array}{c}\text { MVP time } \\
(\mathrm{s})\end{array}$ & $\begin{array}{c}\text { Iteration } \\
\text { number }\end{array}$ & $\begin{array}{c}\text { Total time } \\
(\mathrm{s})\end{array}$ \\
\hline A & 1,893 & 1,262 & 2.7 & 21.5 & 59.1 & 4.2 & 87.5 & 0.15 & 25 \\
B & 7,992 & 5,328 & 10.2 & 90.7 & 88.6 & 8.4 & 197.9 & 0.6 & 36 & 22.8 \\
C & 33,330 & 22,220 & 43.5 & 378.3 & 118.2 & 12.6 & 552.6 & 2.2 & 52 & 118.4 \\
D & 139,002 & 92,668 & 178 & 1,541 & 147.8 & 16.8 & $1,883.6$ & 8.7 & 74 & 655.8 \\
E & 563,292 & 375,528 & 727.9 & 6,245 & 177.36 & 21 & $7,170.4$ & 31.2 & 110 & 3,480 \\
F & $2,301,804$ & $1,534,536$ & $2,932.9$ & 25,518 & 206.9 & 25.2 & 28,683 & 120.9 & 174 & $21,232.6$ \\
\hline
\end{tabular}

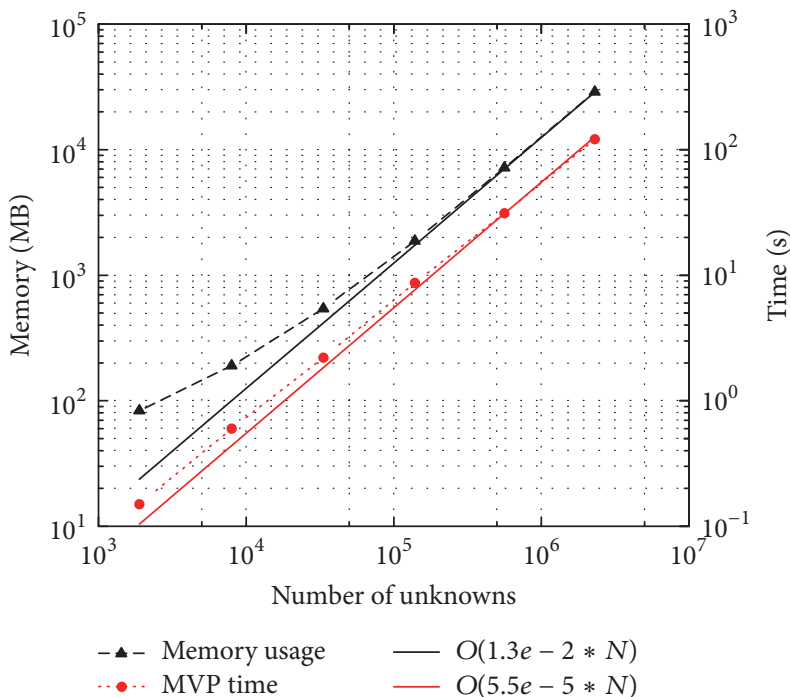

Figure 2: CPU time for each MVP and memory usage in the example of the PEC cube.

The CPU times for each MVP and the memory usage is plotted in Figure 2. It can be found that the computational complexity and the memory requirement of MLCSB-AEFIE both scale as $O(N)$, where $N$ are the unknown numbers. The detailed computational statistics are summarized in Table 1, and the iteration histories are shown in Figure 3 for comparison. By using the preconditioner in [9], the iteration converges quickly to $10^{-4}$. A good agreement of the bistatic radar cross sections (RCS) of the five cases is demonstrated in Figure 4.

4.3. Error Analysis. In the proposed MLCSB-AEFIE, the numerical error is mainly from expansion and aggregation. Since the expansion and aggregation processes are similar for the vector and scalar potentials, we only show the scalar potential case in the following. For the expansion, the relative errors related to a group with the size of $0.005 \mathrm{~m}$ and average discretization length of $0.001 \mathrm{~m}$ are studied. The relative error of expansion is defined as

$$
e=\frac{\left|P(\mathbf{r})-P^{\mathrm{CSB}}(\mathbf{r})\right|}{|P(\mathbf{r})|},
$$

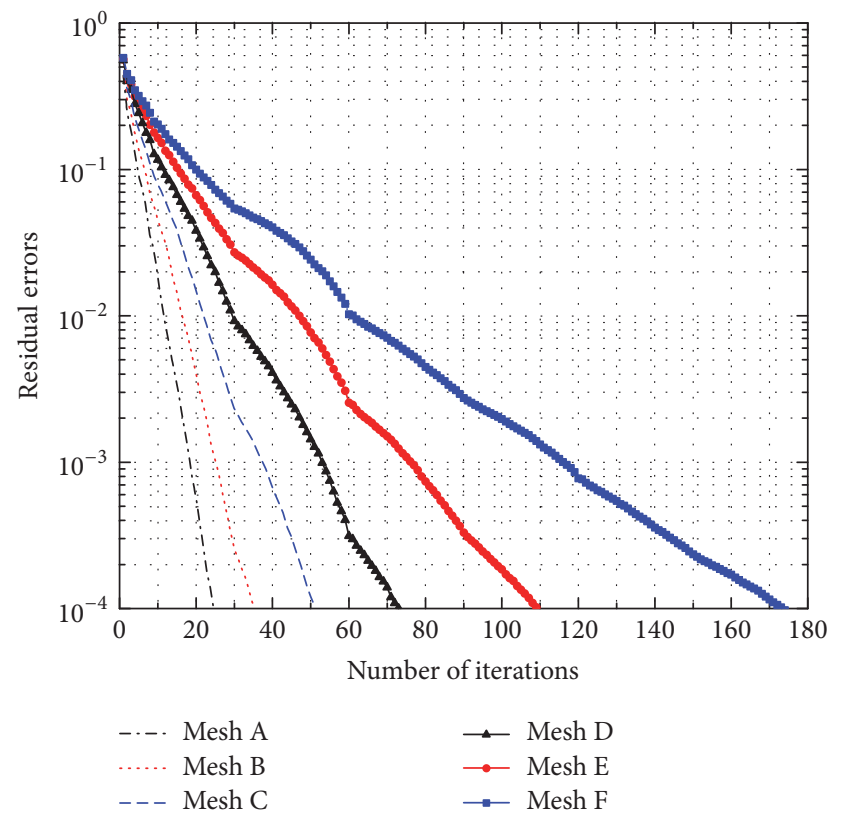

FIGURE 3: Iteration history of the PEC cube for different meshes.

where $P^{\mathrm{CSB}}(\mathbf{r})$ is the scalar potential obtained via the expansion process in (17), $P(\mathbf{r})$ is the exact data calculated by (15), and the observation point $\mathbf{r}$ is fixed at the center of the nearest cousin group. Figure 5 shows the relative errors $e$ for different numbers of CSBs $Q=30,42,58$, and 82 with respect to the frequencies from $30 \mathrm{~Hz}$ to $300 \mathrm{MHz}$. It is noticed that the expansion errors are almost constant for the same $Q$ in a very wide frequency range, indicating an excellent stability and scalability of the proposed method for broadband computations.

To investigate the interpolation error in the aggregation process, we compare the scalar potentials related to a group with the size of $0.005 \mathrm{~m}$ and the parent group with the size of $0.01 \mathrm{~m}$. The scalar potentials are calculated by CSBs, while the CSB coefficients of the parent group are aggregated from the child group. The RMS relative error is shown in Figure 6, which is defined as

$$
\operatorname{RMS}(P)=\sqrt{\frac{1}{N} \sum_{i=1}^{N} \frac{\left\|P_{l}^{\mathrm{CSB}}\left(\mathbf{r}_{i}\right)-P_{l+1}^{\mathrm{CSB}}\left(\mathbf{r}_{i}\right)\right\|^{2}}{\left\|P_{l+1}^{\mathrm{CSB}}\left(\mathbf{r}_{i}\right)\right\|^{2}}}
$$




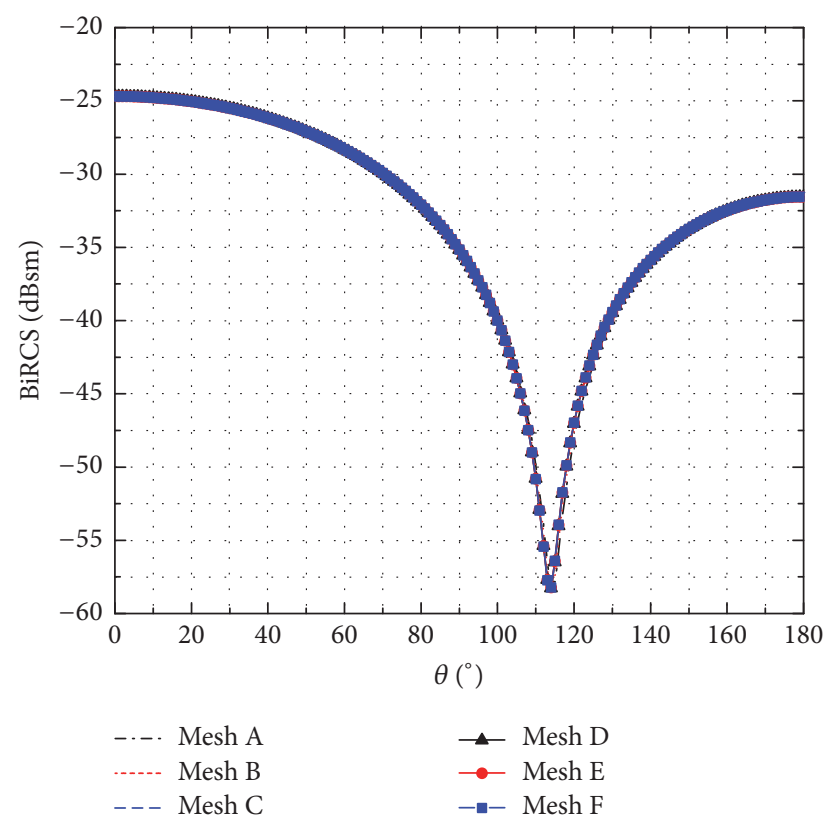

FIGURE 4: Bistatic RCS of the PEC cube with different meshes.

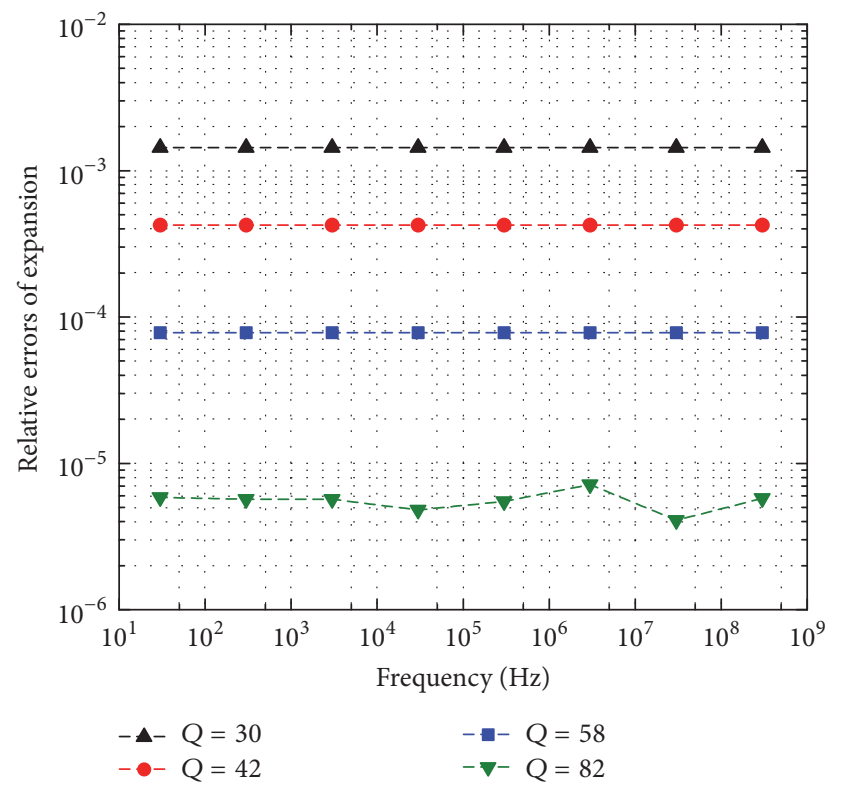

FIGURE 5: Relative errors of expansion.

and here $P_{l}^{\mathrm{CSB}}\left(\mathbf{r}_{i}\right), P_{l+1}^{\mathrm{CSB}}\left(\mathbf{r}_{i}\right)$ are the scalar potentials of the parent group and the child group, respectively, and $\mathbf{r}_{i}$ is set on azimuth circle centered at the parent group. Figure 6 also shows a constant accuracy level for different frequencies with the same $Q$.

4.4. Scattering of Multiscale Cone. The performance of the MLCSB-AEFIE for multiscale structures is evaluated by a nonuniformly meshed cone illustrated in Figure 7. The radius of the cone is $5 \mathrm{~m}$ at the bottom and the height is $10 \mathrm{~m}$. We mesh the cone with a length of $0.1 \mathrm{~m}$ at the bottom and gradually reduce it to $0.001 \mathrm{~m}$ at the sharp

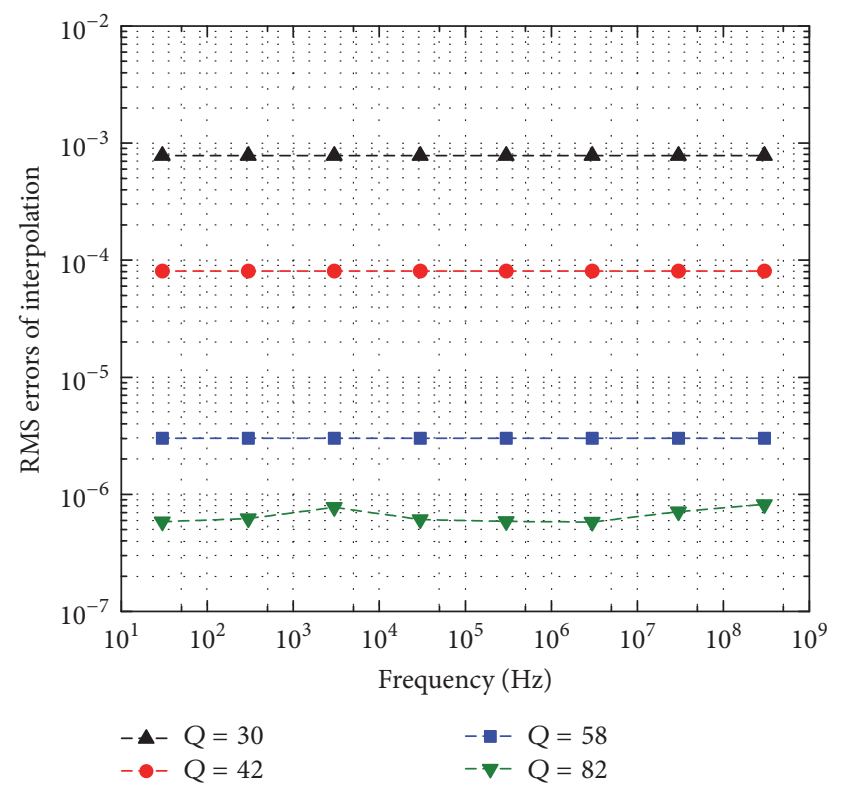

FIGURE 6: RMS errors of interpolation.

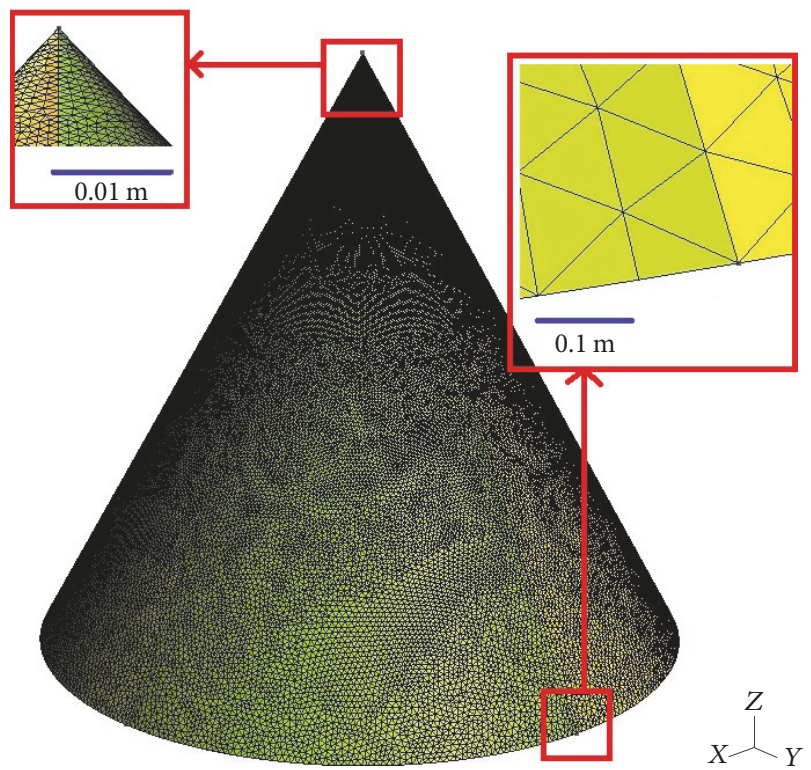

FIGURE 7: A cone of radius $5 \mathrm{~m}$ and height $10 \mathrm{~m}$, which is meshed with average length $0.1 \mathrm{~m}$ at the bottom and $0.001 \mathrm{~m}$ at the sharp point. Mesh details are shown with reference lengths $0.1 \mathrm{~m}$ and $0.01 \mathrm{~m}$.

point. Finally, the cone is discretized with 191,976 planar patches, and hence 287,964 interior edges. Figure 8 shows the bistatic RCS of the cone excited by a $y$-polarized plane wave incident from the $x$ direction at $10 \mathrm{KHz}$ and $10 \mathrm{MHz}$. In this example, MLCSB-AEFIE converges to relative residual error of $10^{-3}$ within 73 and 67 iterations, for $10 \mathrm{KHz}$ and $10 \mathrm{MHz}$, respectively. 


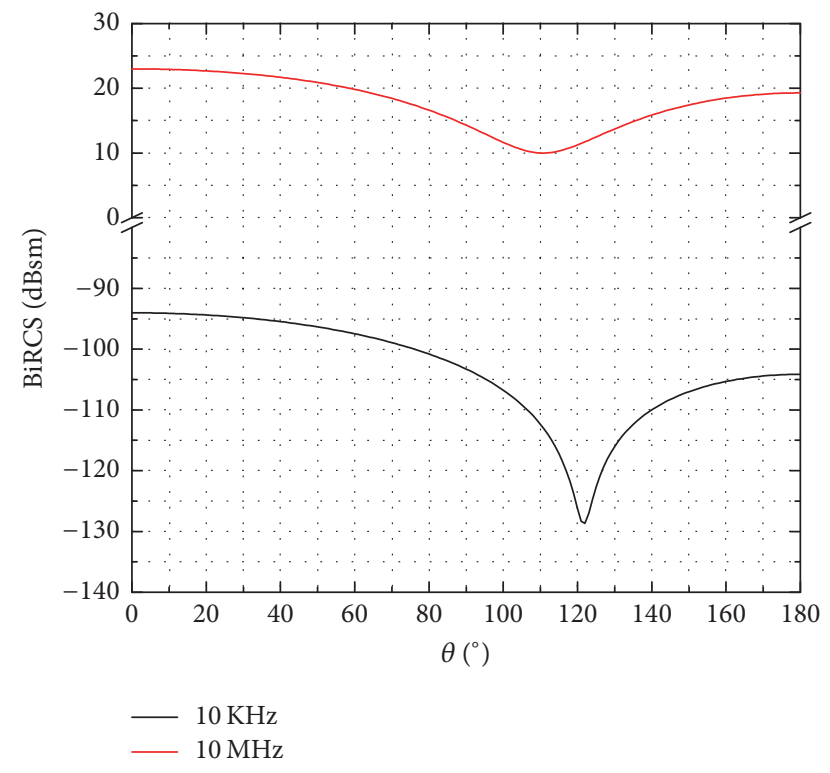

FIgURE 8: Bistatic RCS of the PEC cone at $10 \mathrm{KHz}$ and $10 \mathrm{Mhz}$.

\section{Conclusion}

In this paper, we have proposed a MLCSB-AEFIE method for the well-known low-frequency problem. The vector and scalar potentials from the current and charge unknowns are expended with CSBs. An aggregation matrix is obtained for the CSB expansion coefficients to form an efficient multilevel algorithm. Numerical examples have validated the good accuracy, efficiency, and scalability of the proposed method for low-frequency problems.

\section{Conflicts of Interest}

The authors declare that there are no conflicts of interest regarding the publication of this paper.

\section{Acknowledgments}

This work is supported partly by the National Excellent Youth Fund by NSFC no. 61425010, Programme of Introducing Talents of Discipline to Universities under Grant no. b07046, the Chang Jiang Scholar Project of MOE, and the National Natural Science Foundation of China under Grant no. 61490695 .

\section{References}

[1] R. F. Harrington and J. L. Harrington, Field Computation by Moment Methods, Oxford University Press, Oxford, UK, 1996.

[2] Z. G. Qian and W. C. Chew, "A quantitative study on the low frequency breakdown of EFIE," Microwave and Optical Technology Letters, vol. 50, no. 5, pp. 1159-1162, 2008.

[3] F. P. Andriulli, "Loop-star and loop-tree decompositions: analysis and efficient algorithms," IEEE Transactions on Antennas and Propagation, vol. 60, no. 5, pp. 2347-2356, 2012.
[4] S. Yan, J.-M. Jin, and Z. Nie, "EFIE analysis of low-frequency problems with loop-star decomposition and Calderón multiplicative preconditioner," IEEE Transactions on Antennas and Propagation, vol. 58, no. 3, pp. 857-867, 2010.

[5] M. Taskinen and P. Yla-Oijala, "Current and charge integral equation formulation," IEEE Transactions on Antennas and Propagation, vol. 54, no. 1, pp. 58-67, 2006.

[6] D. Gope, A. Ruehli, and V. Jandhyala, "Solving low-frequency EM-CKT problems using the PEEC method," IEEE Transactions on Advanced Packaging, vol. 30, no. 2, pp. 313-320, 2007.

[7] A. Das and D. Gope, "Modified SPIE formulation for lowfrequency stability of electric field integral equation," in Proceedings of the 5th IEEE Applied Electromagnetics Conference (AEMC '15), Guwahati, India, December 2015.

[8] Z. G. Qian and W. C. Chew, "An augmented electric field integral equation for high-speed interconnect analysis," Microwave and Optical Technology Letters, vol. 50, no. 10, pp. 2658-2662, 2008.

[9] Z.-G. Qian and W. C. Chew, "Fast full-wave surface integral equation solver for multiscale structure modeling," IEEE Transactions on Antennas and Propagation, vol. 57, no. 11, pp. 35943601, 2009.

[10] Y. P. Chen, L. Jiang, Z.-G. Qian, and W. C. Chew, "Modeling electrically small structures in layered medium with augmented EFIE method," in Proceedings of the IEEE International Symposium on Antennas and Propagation and USNC/URSI National Radio Science Meeting, APSURSI 2011, pp. 3218-3221, Spokane, Wash, USA, July 2011.

[11] Y. G. Liu, W. C. Chew, L. Jiang, and Z. Qian, "A memory saving fast A-EFIE solver for modeling low-frequency largescale problems," Applied Numerical Mathematics, vol. 62, no. 6, pp. 682-698, 2012.

[12] Z.-G. Qian and W. C. Chew, "Enhanced A-EFIE with perturbation method," IEEE Transactions on Antennas and Propagation, vol. 58, no. 10, pp. 3256-3264, 2010.

[13] Z.-H. Ma, L. J. Jiang, and W. C. Chew, "Loop-tree free augmented equivalence principle algorithm for low-frequency problems," Microwave and Optical Technology Letters, vol. 55, no. 10, pp. 2475-2479, 2013.

[14] K.-J. Xu, X.-M. Pan, and X.-Q. Sheng, "An augmented EFIE with discontinuous Galerkin discretization," in Proceedings of the IEEE International Conference on Computational Electromagnetics, ICCEM 2016, pp. 106-108, Guangzhou, China, February 2016.

[15] J. S. Zhao and W. C. Chew, "Applying LF-MLFMA to solve complex PEC structures," Microwave and Optical Technology Letters, vol. 28, no. 3, pp. 155-160, 2001.

[16] Y. Zheng, Y. Zhao, Z. Nie, and Q. Cai, "Full-wave fast solver for circuit devices modeling," Applied Computational Electromagnetics Society Journal, vol. 30, no. 10, pp. 1115-1121, 2015.

[17] D. Z. Ding, Y. Shi, Z. N. Jiang, and R. S. Chen, "Augmented EFIE with adaptive cross approximation algorithm for analysis of electromagnetic problems," International Journal of Antennas and Propagation, vol. 2013, Article ID 487276, 9 pages, 2013.

[18] M. M. Jia, S. Sun, and W. C. Chew, "Accelerated A-EFIE with perturbation method using fast fourier transform," in Proceedings of the IEEE Antennas and Propagation Society International Symposium (APSURSI '14), pp. 2148-2149, Memphis, Tenn, USA, July 2014.

[19] K. Tap, P. H. Pathak, and R. J. Burkholder, "Complex source beam-moment method procedure for accelerating numerical 
integral equation solutions of radiation and scattering problems," IEEE Transactions on Antennas and Propagation, vol. 62, no. 4, part 2, pp. 2052-2062, 2014.

[20] K. Tap, Complex source point beam expansions for some electromagnetic radiation and scattering problems [Ph.D. thesis], The Ohio State University, Columbus, Ohio, USA, 2007.

[21] E. Martini and S. Maci, "Generation of complex source point expansions from radiation integrals," Progress in Electromagnetics Research, vol. 152, no. 3, pp. 17-31, 2015.

[22] Z. H. Fan, X. Hu, and R. S. Chen, "Multilevel complex source beam method for electromagnetic scattering problems," IEEE Antennas and Wireless Propagation Letters, vol. 14, pp. 843-846, 2015.

[23] K. C. Wang, Z. H. Fan, M. M. Li, and R. S. Chen, "An effective MoM Solution with nested complex source beam method for electromagnetic scattering problems," IEEE Transactions on Antennas and Propagation, vol. 64, no. 6, pp. 2546-2551, 2016.

[24] T. B. Hansen and G. Kaiser, "Huygens' principle for complex spheres," IEEE Transactions on Antennas and Propagation, vol. 59, no. 10, pp. 3835-3847, 2011.

[25] Ö. Ergül and L. Gürel, The Multilevel Fast Multipole Algorithm (MLFMA) for Solving Large-Scale Computational Electromagnetics Problems, John Wiley \& Sons, Hoboken, NJ, USA, 2014. 


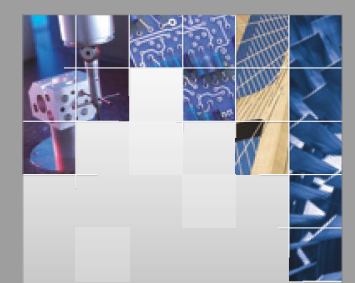

\section{Enfincering}
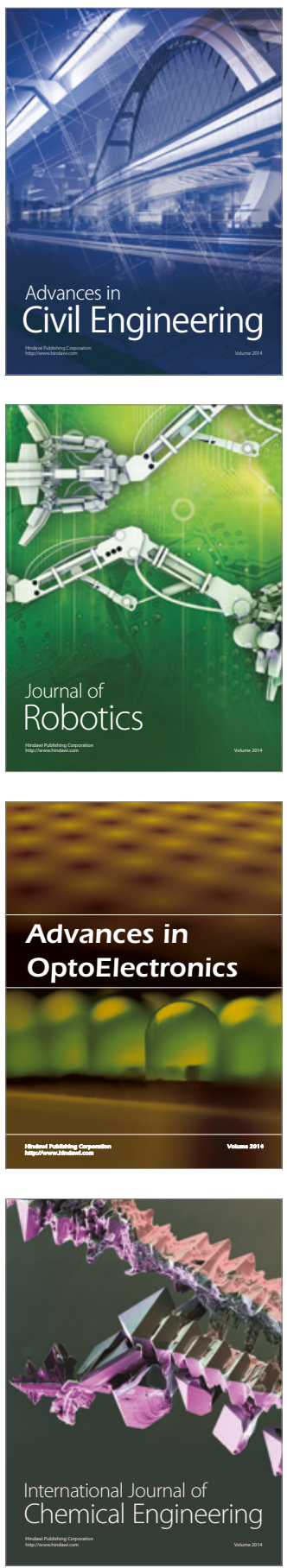

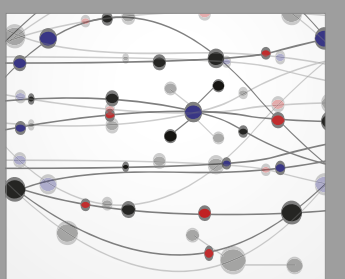

The Scientific World Journal

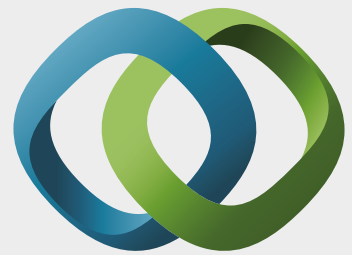

\section{Hindawi}

Submit your manuscripts at

https://www.hindawi.com
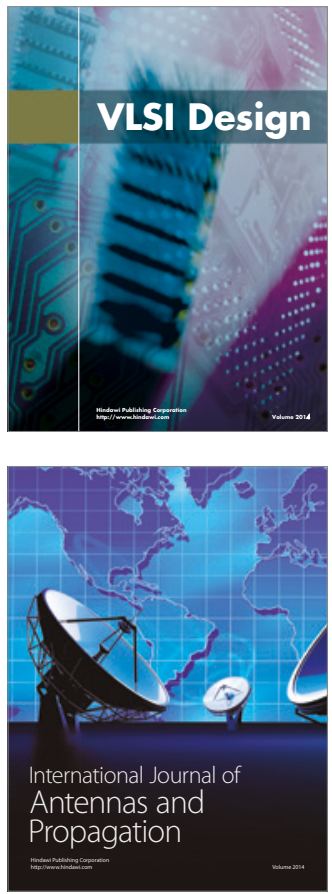

\section{Rotating}

Machinery
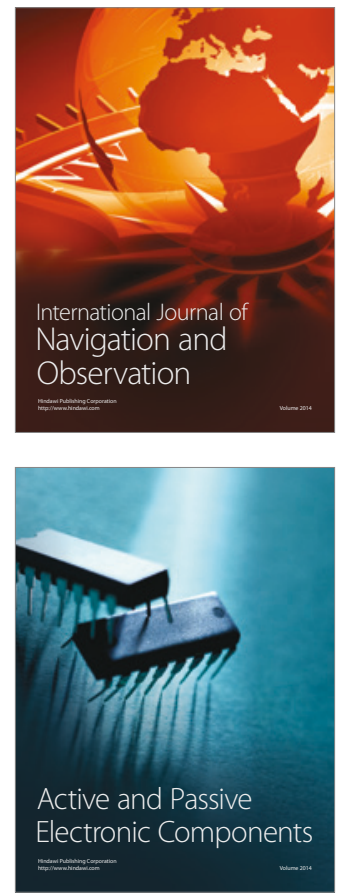
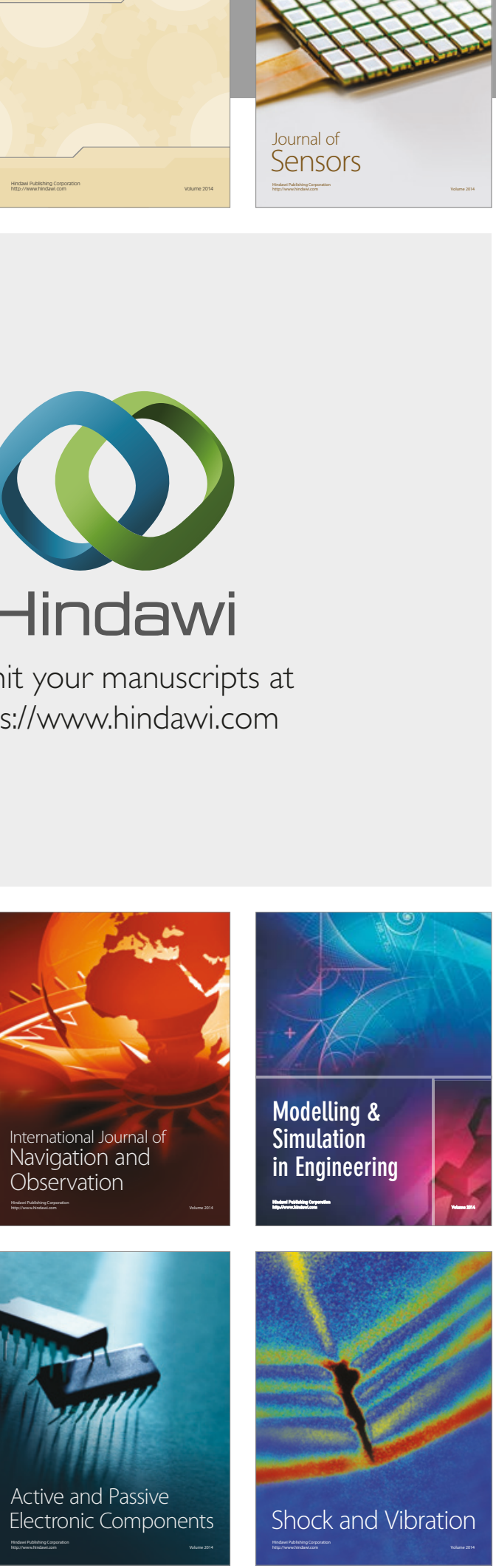
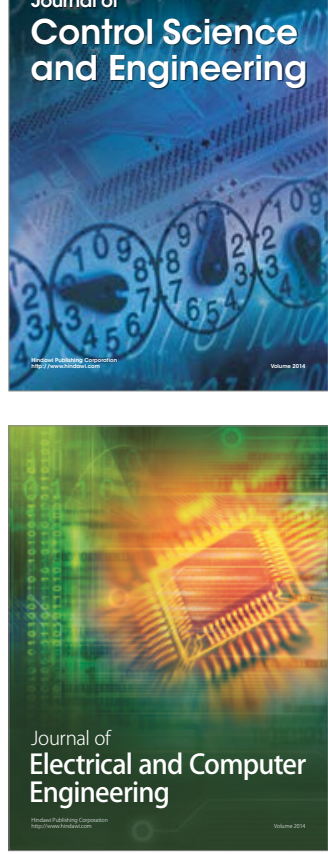

Distributed

Journal of

Control Science

and Engineering
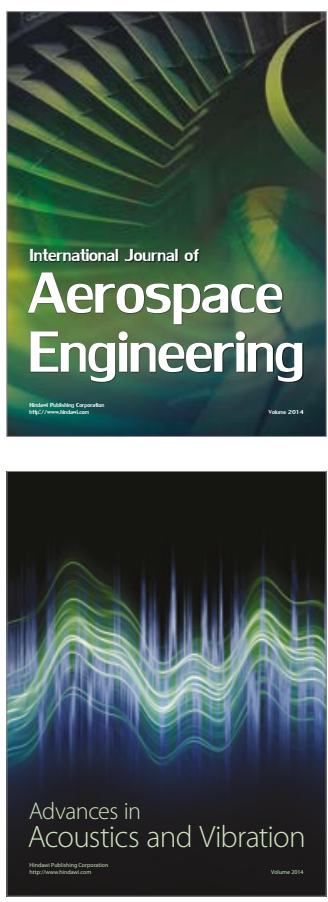

Sensor Networks 